\title{
The Four Different Types of Internal Hernia Occurring After Laparascopic Roux-en-Y Gastric Bypass Performed for Morbid Obesity: Are There Any Multidetector Computed Tomography (MDCT) Features Permitting Their Distinction?
}

\author{
Aida Kawkabani Marchini • Alban Denys • \\ Alexandre Paroz • Sébastien Romy • Michel Suter • \\ Nicolas Desmartines $\cdot$ Reto Meuli • Sabine Schmidt
}

Published online: 12 February 2011

(C) Springer Science+Business Media, LLC 2011

\begin{abstract}
Background Four different types of internal hernias $(\mathrm{IH})$ are known to occur after laparoscopic Roux-en-Y gastric bypass (LRYGBP) performed for morbid obesity. We evaluate multidetector row helical computed tomography (MDCT) features for their differentiation.

Methods From a prospectively collected database including 349 patients with LRYGBP, 34 acutely symptomatic patients (28 women, mean age 32.6 ), operated on for $\mathrm{IH}$ immediately after undergoing MDCT, were selected. Surgery confirmed $4(11.6 \%)$ patients with transmesocolic, 10 (29.4\%) with Petersen's, 15 (44.2\%) with mesojejunal, and $5(14.8 \%)$ with jejunojejunal $\mathrm{IH}$. In consensus, 2 radiologists analyzed 13 MDCT features to distinguish the four types of IH. Statistical significance was calculated $(p<$ 0.05 , Fisher's exact test, chi-square test).

Results MDCT features of small bowel obstruction (SBO) $(n=25,73.5 \%)$, volvulus $(n=22,64.7 \%)$, or a cluster of small bowel loops (SBL) $(n=27,79.4 \%)$ were inconsistently present and overlapped between the four IH. The following features allowed for IH differentiation: left upper quadrant clustered small bowel loops $(p<0.0001)$ and a mesocolic hernial orifice $(p=0.0003)$ suggested transmeso-
\end{abstract}

A. Kawkabani Marchini $\cdot$ A. Denys $\cdot$ R. Meuli $\cdot$ S. Schmidt $(\bowtie)$ Department of Radiology, University Hospital-CHUV,

1011 Lausanne, Switzerland

e-mail: sabine.schmidt@chuv.ch

A. Paroz $\cdot$ S. Romy $\cdot$ M. Suter $\cdot$ N. Desmartines

Department of Visceral Surgery, University Hospital-CHUV,

Lausanne, Switzerland colic IH. SBL abutting onto the left abdominal wall ( $p=$ 0.0021 ) and left abdominal shift of the superior mesenteric vessels (SMV) $(p=0.0045)$ suggested Petersen's hernia. The SMV predominantly shifted towards the right anterior abdominal wall in mesojejunal hernia $(p=0.0033)$. Location of the hernial orifice near the distal anastomosis $(p=0.0431)$ and jejunojejunal suture widening $(p=0.0005)$ indicated jejunojejunal hernia.

Conclusions None of the four IH seems associated with a higher risk of SBO. Certain MDCT features, such as the position of clustered SBL and hernial orifice, help distinguish between the four IH and may permit straightforward surgery.

Keywords CT — computed tomography - Bariatric surgery . Internal hernia $\cdot$ Small bowel obstruction

\section{Introduction}

Bariatric surgery is recognized as the only effective treatment for morbid obesity. The various forms of bariatric surgery include volume restriction (vertical banding), interventions causing malabsorption (jejunoileal bypass), and procedures that combine both, namely Roux-en-Y gastric bypass (RYGBP) [1, 2]. RYGBP has been shown to be the most effective technique with a reported weight loss of $60-70 \%$ over a period of 10 years follow-up [3, 4]. Therefore, RYGBP has now become the most commonly performed weight loss operation worldwide $[5,6]$. 
Compared with the traditionally performed "open" surgery, the minimally invasive alternative, the laparoscopic Roux-en-Y gastric bypass (LRYGBP), has gained far wider acceptance because of fewer wound complications, shorter hospital stays, and better cosmetic results [7-9]. Likewise, postoperative intra-abdominal adhesions are less common. However, LRYGBP is associated with a relatively high incidence $(0.8-5 \%)$ of internal hernias $(\mathrm{IH})$, unlike open RYGBP [4, 6-8, 10-13]. IH has even been reported to be the most frequent cause of small bowel obstruction (SBO) occurring after LRYGBP [14].

It has been postulated that the weight loss seen in these very obese patients, typically occurring some months after bariatric surgery, causes rapid reduction of the intraperitoneal fat which, in turn, leads to a subsequent enlargement of the surgically created mesenteric defects and a consecutive loosening of the mesenteric sutures $[7,15,16]$. This gives way to the prolapse of small bowel loops into these newly opened windows, the potential IH sites. Secondly, this incidence of IH may also be due to the laparoscopic approach itself. The time interval between LRYGBP and the onset of symptoms indicative of $\mathrm{IH}$ is highly variable, occurring at any time after surgery $[8,11,12]$. IH may lead to severe complications, like small bowel obstruction, possibly followed by bowel ischemia, and even death [17].

The surgical defects leading to the prolapse of small bowel loops have been known to occur at four different sites: either at the incised transverse mesocolon; at the Petersen's space, which represents an opening between the mesocolon and the Roux limb mesentery; at the jejunojejunostomy mesenteric defect, known as mesojejunal hernia; or, finally, at the suture of the two jejunal loops, the biliopancreatic and the Roux limb, called jejunojejunal hernia $[3,4,6-12,16-18]$.

In patients with acute abdominal pain and/or clinical signs of SBO, multidetector computed tomography (MDCT) has become the imaging modality of choice to reveal complications of LRYGBP. MDCT is able to demonstrate intraluminal (bleeding, intussusception) and extraluminal (fluid collections, abscesses) intestinal complications, as well as to point out potential IH. MDCT features of these four IH have only sporadically been described, consisting of small series with potentially redundant radiological signs from one type of $\mathrm{IH}$ to another [2,10,19-21]. The distinction between the individual types by means of MDCT has not yet been attempted. The successful preoperative differentiation would guide the surgeon and allow for the straightforward laparoscopic closure of these defects, thus potentially shortening the intervention. The aim of our study is therefore to evaluate the diagnostic value of different MDCT features that may help distinguish these four IH after LRYGBP.

\section{Material and Methods}

\section{Study Population}

The study received approval from the institutional ethics committee. The review of our prospectively collected computerized surgical database from 2004 to 2008 yielded 349 patients operated on LRYGBP at our and three nearby regional institutions. The surgical procedure of LRYGBP was performed by three experienced surgeons specializing in bariatric surgery (Fig. 1). After the creation of a 10-15-ml gastric pouch along the lesser curvature that was separated from the rest of the stomach, the jejunum was divided approximately $30 \mathrm{~cm}$ distally from the angle of Treitz, mobilized from the mesentery, and brought up to create a side-to-side gastrojejunostomy. This anastomosed jejunal loop has been called the Roux (or alimentary) limb. It was placed retrocolic through an opening created in the transverse mesocolon, or antecolic in front of the transverse colon. The other separate limb, composed of the excluded stomach, the duodenum, and the proximal jejunum, was named the biliopancreatic limb. Finally, a jejunojejunostomy was created (at approximately $30 \mathrm{~cm}$ distally from the Treitz ligament) joining the Roux limb (of about $100-150 \mathrm{~cm}$ of length) and the biliopancreatic limb, using a metallic staple line. Routinely, all mesenteric defects were closed at the initial operation.

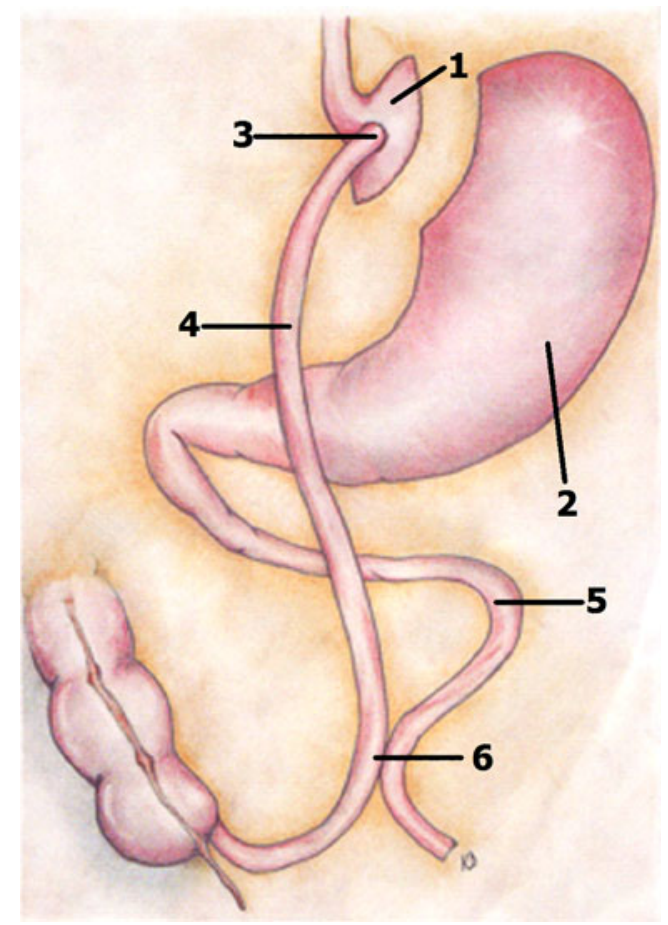

Fig. 1 Schematic drawing of the Roux-en-Y gastric bypass (RYGBP) anatomy: lesser curvature (1), stomach (2), side-to-side gastrojejunostomy (3), Roux (or alimentary) limb (4), biliopancreatic limb (5), side-to-side jejunojejunostomy (6) 
Seventy-one $(4.9 \%)$ out of the 349 patients $(4.9 \%)$ had developed surgically proven internal hernias (IH). Among this group, we selected 34 patients (28 women, mean age 32.6 years, range $27-55$ years of age) with the following inclusion criteria: MDCT had been performed within $48 \mathrm{~h}$ of surgery (mean delay 1.19 days) and the clinical/ radiological data were complete. We excluded 20 patients, who had not undergone MDCT within the $48 \mathrm{~h}$ preceding the surgical intervention, and 17 patients who lack clinical and/or radiological data.

Among our study population of 34 patients, 31 had a retrocolic placement and three others had an antecolic placement of the Roux limb. The mean body mass index in these 34 patients was 43.63 (range 35-59.3) before LRYGBP and had decreased to 27.91 (range 22-47.6) by the time of surgery for $\mathrm{IH}$. The mean delay between LRYGBP and surgery for IH was 28.7 months (range 184 months). Surgery confirmed $4(11.6 \%)$ patients with transmesocolic IH, 10 (29.4\%) with Petersen's, 15 (44.2\%) with mesojejunal, and $5(14.8 \%)$ patients with jejunojejunal $\mathrm{IH}$, craniocaudally numbered according to the position of the hernial orifice in Fig. 2 and also shown on intraoperative photos in Fig. 3.

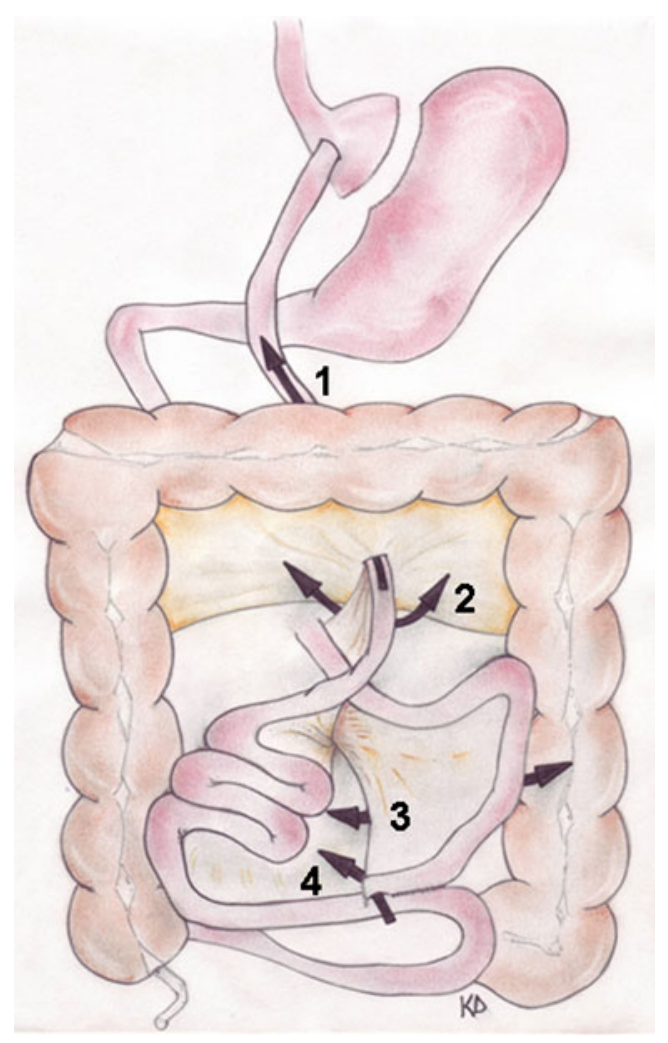

Fig. 2 Schematic drawing localizing the four types of IH occurring after LRYGBP numbered in craniocaudal order according to the position of the hernial orifice. Transmesocolic hernia (1), Petersen's hernia (2), mesojejunal hernia (3), jejunojejunal hernia (4)
In all patients, the mesenteric defects causing the $\mathrm{IH}$ were consequently closed. In seven patients $(20.6 \%)$, further mesenteric defects, without containing small bowel loops, were intraoperatively found and consecutively sutured.

Image Acquisition

Using 16- and 64-slice CT machines, axial image acquisition was performed during venous phase (delay $70 \mathrm{~s})$ according to a standardized protocol. Only the slice thickness varied $(1-5 \mathrm{~mm})$. In 31 patients $(86.1 \%)$, thin $(\leq 2.5 \mathrm{~mm})$ axial slices were reconstructed, and then completed by coronal/sagittal reformatted images. We thus took advantage from the technique of multidetector CT allowing for rapid multiplanar imaging with improved spatial resolution compared to single slice CT and permitting straightforward identification of the anatomic landmarks and the hernial orifices. All patients intravenously received iodinated contrast agent (volume in milliliters=body weight $+30 \mathrm{ml}$, total $\leq 100 \mathrm{ml}, 300 \mathrm{mg} \mathrm{I} / \mathrm{ml}$, Accupaque $^{\circledR}$ ) at a flow rate of $2-3 \mathrm{ml} / \mathrm{s}$, unless contraindicated because of the patient's known allergy $(n=1)$. Before MDCT, 100-200 $\mathrm{ml}$ of diluted hydrosoluble and iodinated contrast medium (Gastrografin ${ }^{\circledR}$ ) was orally administrated, whenever tolerated by the patients $(n=25$, $69.4 \%)$.

\section{Image Analysis}

The images were retrospectively reviewed on a dedicated work station except one patient in whom only hard copy MDCT images were available for the review. In consensus, two abdominal radiologists (12 and 5 years of practical experience in gastrointestinal imaging) analyzed axial and coronal images of each patient. At the time of the review, they were blinded to the specific type of $\mathrm{IH}$, proven by surgery.

The image reading method first consisted in identifying the surgical landmarks, i.e., the Roux and biliopancreatic limb as well as the gastrojejunal and jejunojejunal anastomosis, and in recording their position. In case of retrocolic placement of the Roux limb, we tried to localize the mesocolic window radiologically. The metallic stapler line served as a landmark for the localization of the jejunojejunal suture.

As Table 1 shows, MDCT features of three of the four different types of IH have already been described, mostly sporadically and including few patients only. After reviewing these previous publications $[2,3,5-7,9-11,20,21]$, we divided our analysis into MDCT features indicating SBO and indicating $\mathrm{IH}$ for the distinction between the four types of internal hernias (Table 1). 

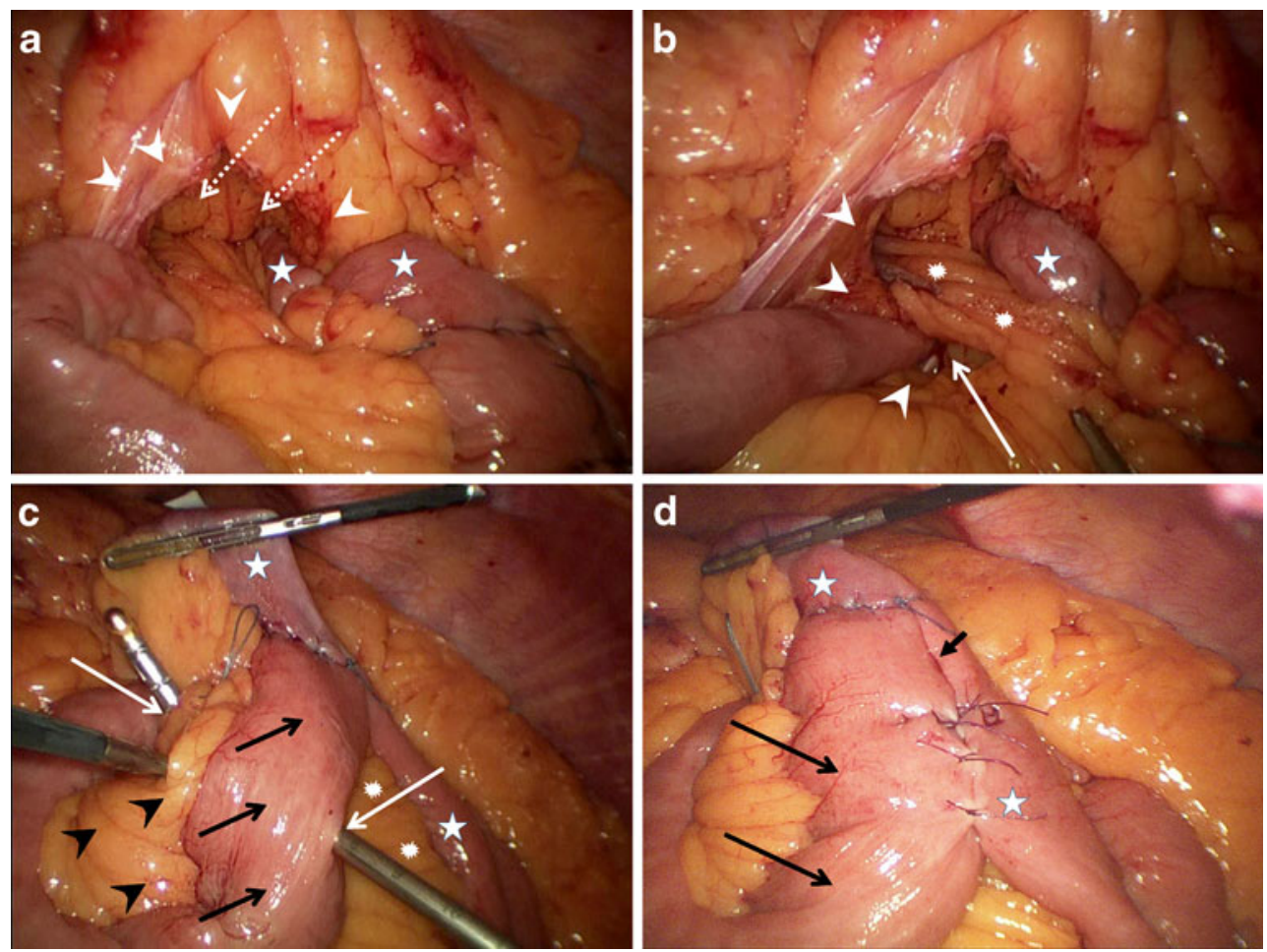

Fig. 3 Laparoscopic view of the four surgically created windows: a transmesocolic window (dotted arrows) surrounded by the incised transverse mesocolon (white arrowheads), thus allowing for the passage of the ascending Roux limb (white stars). b Petersen's window (white long arrow) defined by the transverse mesocolon (white arrowheads) posteriorly and the mesojejunum (white dots) of the Roux limb (white star) anteriorly. It is located just caudally to the Roux limb's passage through the transmesocolic window. c Cranio-

caudal view of the mesojejunal window (white long arrows) indicated by a passing forceps: it is defined by the mesojejunum (black arrowheads) of the biliopancreatic loop (black long arrows) lying anteriorly and the mesojejunum (white dots) of the Roux limb (white stars) lying posteriorly. d Craniocaudal view of the jejunojejunal window (black short arrow) located between the Roux limb (white stars) and the biliopancreatic loop (black long arrows)

Table 1 Previously reported MDCT features in relation with the four types of internal hernias

\begin{tabular}{|c|c|c|c|c|c|}
\hline MDCT features & $\begin{array}{l}\text { Occuring in any of } \\
\text { these four IH }\end{array}$ & Transmesocolic IH & Petersen's IH & Mesojejunal IH & Jejunojejunal IH \\
\hline Dilated excluded stomach & & & & [11] & \\
\hline Dilated Roux limb & & {$[2,11]$} & & {$[11,21]$} & \\
\hline \multicolumn{6}{|l|}{ Displaced distal anastomosis } \\
\hline To the right & {$[5,9,11]$} & & & & \\
\hline SB obstruction & {$[5,9-11]$} & & {$[10,11,20]$} & & {$[6]$} \\
\hline Point of transition & [7] & & & & \\
\hline Volvulus (whirl sign) & {$[5,9]$} & & & {$[11,21]$} & {$[6]$} \\
\hline Cluster of small bowel loops & {$[5,7,9,11]$} & {$[11]$} & & & \\
\hline \multicolumn{6}{|l|}{ Location of clustered SB loops } \\
\hline $\begin{array}{l}\text { Left upper abdomen (posterior to } \\
\text { stomach) }\end{array}$ & {$[3,7,11]$} & {$[2,3,7,10,20,21]$} & & & \\
\hline Central middle abdomen (anterior wall) & {$[7,11]$} & & & [7] & \\
\hline $\begin{array}{l}\text { Right paraumbilical abdomen } \\
\text { (anterolateral wall) }\end{array}$ & {$[11]$} & & & [21] & \\
\hline Left middle abdomen (anterolateral wall) & {$[7,11]$} & & {$[3,21]$} & & \\
\hline Altered course of SMA/SMV & {$[5,7]$} & {$[2,20]$} & {$[7,10,20,21]$} & {$[21]$} & {$[6]$} \\
\hline SB loops behind SMA/SMV & {$[5,9]$} & & & & \\
\hline
\end{tabular}

$S B$ small bowel, $S M A$ superior mesenteric artery, $S M V$ superior mesenteric vein 
MDCT signs suggestive of SBO were the dilatation of the excluded stomach, the Roux limb, biliopancreatic limb, or the jejunojejunal anastomosis. Small bowel dilatation was taken into account, whenever the intestinal lumen measured $\geq 3 \mathrm{~cm}$ in diameter. SBO was defined by an abrupt caliber change with distal collapsed small bowel loops, possibly with a beak sign at the transition point, the latter also being recorded. Small bowel volvulus was defined by the presence of a whirl sign (whirled appearance of mesenteric vessels and fat surrounded by dilated and stretched small bowel loops), vascular engorgement, with fat stranding, free fluid, and possibly intestinal wall thickening $[9,11]$.

Secondly, MDCT signs suggesting an internal hernia were taken into account: the presence and anatomical position of an abnormal cluster of small bowel loops was recorded $[3,7,10]$ (close to the anterior or left/right anterolateral abdominal wall without any fat interposition, anterior/posterior to the Roux limb), and presence and position of an hernial orifice (left/right hypochondrium or periumbilical region, close to the jejunojejunal anastomosis or at the level of the mesocolic window) were noted. The possible shift of the jejunojejunal anastomosis was recorded. At our institutions, all jejunojejunal anastomoses had been created in the left hemiabdomen and anterior or lateral displacement close to the left or right abdominal wall suggested torsion of the mesenteric root [5]. The course of the superior mesenteric vessels (midline or deviation to the left/right abdominal cavity) was recorded [21], with the exception of the patient whose MDCT was performed without IV contrast agent injection due to a previous history of contrast medium allergy.

The loosening of the sutures at the distal jejunojejunal anastomosis has been described as the underlying cause for the herniation of small bowel loops into the space between the two jejunal loops, the biliopancreatic and the Roux limb, running parallel at the anastomic site. Thus, the widening between the suture of the side-to-side jejunostomy, and the stapler line used for closing the afferent limb and fixing it at the mesentery, was also included in our analysis [6], whenever identified on MDCT. Finally, the presence of small bowel loops other than the duodenum behind the superior mesenteric artery was noted [5]. The relative frequency of each of these signs occurring in each group of the four IH was calculated statistically.

\section{Statistical Analysis}

Statistical analysis was performed with a JMP 7.0 statistical package (SAS Institute, Cary, NC, USA). The results of our image analysis were correlated with the surgical findings, i.e., one of the four types of IH defined above, accounting for the clinical symptoms. Statistical significance as determined by $p$ values $(p<0.05$ considered to show significance) was calculated applying the Fisher's exact test and, for larger sample size, the chi-square test, whenever required, applied on each of the above defined MDCT findings.

\section{Results}

Table 2 shows the frequency of the selected MDCT features for each type of IH separately and for all $34 \mathrm{IH}$ considered together.

\section{Recognition of the Anatomical Landmarks}

In each of the 34 patients, we could identify the surgical landmarks, i.e., the Roux and biliopancreatic limbs, as well as the gastrojejunal and jejunojejunal anastomosis. All patients with antecolic alimentary limb were identified.

\section{MDCT Features Indicative of SBO}

Signs of SBO were detected in 25 (73.5\%) patients without statistical significance for any type of IH $(p=0.254)$, neither in case of a point of transition $(n=20, p=0.344)$ nor in case of volvulus $(n=22,64.7 \%, p=0.900)$. Dilatation of the excluded stomach $(n=7,20.6 \%, p=0.118)$, of the Roux $\operatorname{limb}(n=9,26.5 \%, p=0.102)$, and/or of the biliopancreatic $\operatorname{limb}(n=9,26.5 \%, p=0.390)$ was inconsistently present and showed a considerable overlap between the four types of $\mathrm{IH}$.

\section{MDCT Features Indicative of IH}

Among the signs indicative of $\mathrm{IH}$, a cluster of small bowel loops was present in $27(79.4 \%)$ patients, inconsistently encountered in all four types of IH without preference ( $p=$ 0.514). The following MDCT features suggested a specific type of IH: In three of our four patients with transmesocolic $\mathrm{IH}$, the internal hernial orifice was identified, always situated at the level of the surgically created mesocolic window ( $p=0.0003$ ), and corresponding to the point of transition from dilated to collapsed SB loops (Fig. 4). In all four patients with transmesocolic hernia, the clustered small bowel loops were situated in the left upper abdomen, between the body of the stomach and the spleen $(p<$ 0.0001) (Fig. 4; Table 2).

In nine out of the ten patients with Petersen's hernia, clustered SB loops were located in the left middle abdomen, abutting upon the left anterolateral abdominal wall $(p=0.0021)$, while the SMV predominantly shifted to the left abdominal cavity $(p=0.0045)$ (Fig. 5). Seven of them $(70 \%)$ had signs of SBO. In seven patients with 
Table 2 Frequency and significance of the 14 MDCT features for differenciating the $4 \mathrm{IH}$

\begin{tabular}{|c|c|c|c|c|c|}
\hline & $\begin{array}{l}\text { Overall frequency } \\
(n=34)\end{array}$ & $\begin{array}{l}\text { Transmesocolic IH } \\
(n=4)\end{array}$ & $\begin{array}{l}\text { Petersen's IH } \\
(n=10)\end{array}$ & $\begin{array}{l}\text { Mesojejunal IH } \\
(n=15)\end{array}$ & $\begin{array}{l}\text { Jejunojejunal IH } \\
(n=5)\end{array}$ \\
\hline Dilated excluded stomach & 7 & 1 & 2 & 3 & 1 \\
\hline Dilated Roux limb & 9 & 2 & 2 & 3 & 2 \\
\hline Dilated biliopancreatic limb & 9 & 1 & 1 & 6 & 1 \\
\hline \multicolumn{6}{|l|}{ Shift of the JJ anastomosis } \\
\hline To the right & 9 & 1 & 5 & 3 & \\
\hline To the left abdominal wall & 7 & 1 & 2 & 2 & 2 \\
\hline Small bowel obstruction & 25 & 4 & 7 & 10 & 4 \\
\hline Volvulus & 22 & 3 & 7 & 9 & 3 \\
\hline Point of transition & 20 & 3 & 4 & 9 & 4 \\
\hline \multicolumn{6}{|l|}{ Location of transition point/hernial orifice } \\
\hline Right upper abdomen & 2 & & & & \\
\hline Left upper abdomen (or mesocolic window) & 4 & $3(p=0.0003)$ & 1 & & \\
\hline Central middle abdomen & 5 & & 3 & 2 & \\
\hline Left middle abdomen (near JJ anastomosis) & 9 & & & 5 & $4(p=0.0431)$ \\
\hline Presence of clustered small bowel (SB) loops & 27 & 4 & 10 & 10 & 3 \\
\hline \multicolumn{6}{|l|}{ Location of clustered SB loops } \\
\hline Left upper abdomen & 4 & $4(p<0.0001)$ & & & \\
\hline Central middle abdomen (anterior wall) & 5 & & 1 & 2 & 2 \\
\hline $\begin{array}{l}\text { Right paraumbilical abdomen } \\
\text { (anterolateral wall) }\end{array}$ & 7 & & & 7 & \\
\hline Left middle abdomen (anterolateral wall) & 11 & & $9(p=0.0021)$ & 1 & 1 \\
\hline Widening of the $\mathrm{JJ}$ anastomosis & 8 & & & 3 & $5(p=0.0005)$ \\
\hline \multicolumn{6}{|l|}{ Altered course of SMV/SMA } \\
\hline To the right & 8 & & & $7(p=0.0033)$ & 1 \\
\hline To the left & 13 & 3 & $8(p=0.0045)$ & 2 & \\
\hline Small bowel loops behind SMV/SMA & 9 & 1 & 2 & 6 & \\
\hline
\end{tabular}

$S M A$ superior mesenteric artery, $S M V$ superior mesenteric vein

mesojejunal hernia, a shift of the proximal part of the superior mesenteric vessels was seen towards the right anterior paraumbilical region $(p=0.0033)$ (Fig. 6), and all these seven patients also showed signs of SBO.

In four of five patients with jejunojejunal hernia, the hernial orifice, corresponding to the transition point of SBO in each case, was located adjacent to the jejunojejunal anastomosis $(p=0.0431)$, that is in the middle left abdomen. The widening of the distal jejunojejunal anastomosis (Fig. 7) was seen in all five patients with jejunojejunal hernia, thus statistically significant $(p=0.0005)$ for this type of $\mathrm{IH}$, since it was detected only in three patients with mesojejunal IH, and never in the two other types. Four out of them had signs of SBO. Neither the shift of the jejunojejunal anastomosis to the right $(n=9)$ or the left $(n=7)$ abdominal wall was associated with a specific type of IH $(p=0.586)$. Finally, the presence of small bowel loops other than the duodenum behind the superior mesenteric artery was detected in nine (26.5\%) patients, but was not significant for any of the four types of $\mathrm{IH}(p=0.423)$.

\section{Discussion}

Our current study assesses MDCT features for each type of IH after LRYGBP. We have included 34 acutely symptomatic patients with surgically proven IH undergoing MDCT within the $48 \mathrm{~h}$ preceding the operation. MDCT signs of SBO due to IH were inconsistently present in all four types. Based on blinded consensus reading, we have tried to assign specific MDCT features to each type of IH.

Clinical symptoms of internal hernia occurring after LRYGBP vary widely, from intermittent abdominal pain to signs of intestinal obstruction. In acutely symptomatic patients, the diagnosis of an IH requires surgical correction in order to avoid potentially fatal mechanical complications with small bowel gangrene and subsequent intestinal perforation [7, 13, 22].

Our study is focused on MDCT signs of IH after LRYGBP. Carucci et al. have described certain features of IH after LRYGB on small bowel follow-through (SBFT) but were not able to find any signs distinguishing the 
Fig. 4 Transmesocolic hernia occurring in a 44-year-old woman 1 month after the LRYBGP. Axial (a, b) and sagittal (c) MDCT images demonstrate retrogastric retrocolic bypass with dilatation of the Roux limb, containing orally administrated hydrosoluble and iodinated contrast medium (a-c black arrow). Note the sac-like cluster of dilated bowel loops (a, c, white dotted arrow) located anteriorly to the spleen and posteriorly to the excluded stomach exerting a mass effect on the posterior wall. Note the point of transition, corresponding to the hernial orifice (b, white arrowhead), located at the level of the surgically created mesocolic orifice, just cranial to the jejunojejunostomy. The transverse mesocolon extends between the transverse colon (TC) and the pancreas $(P)$, nicely seen on the sagittal view (c)
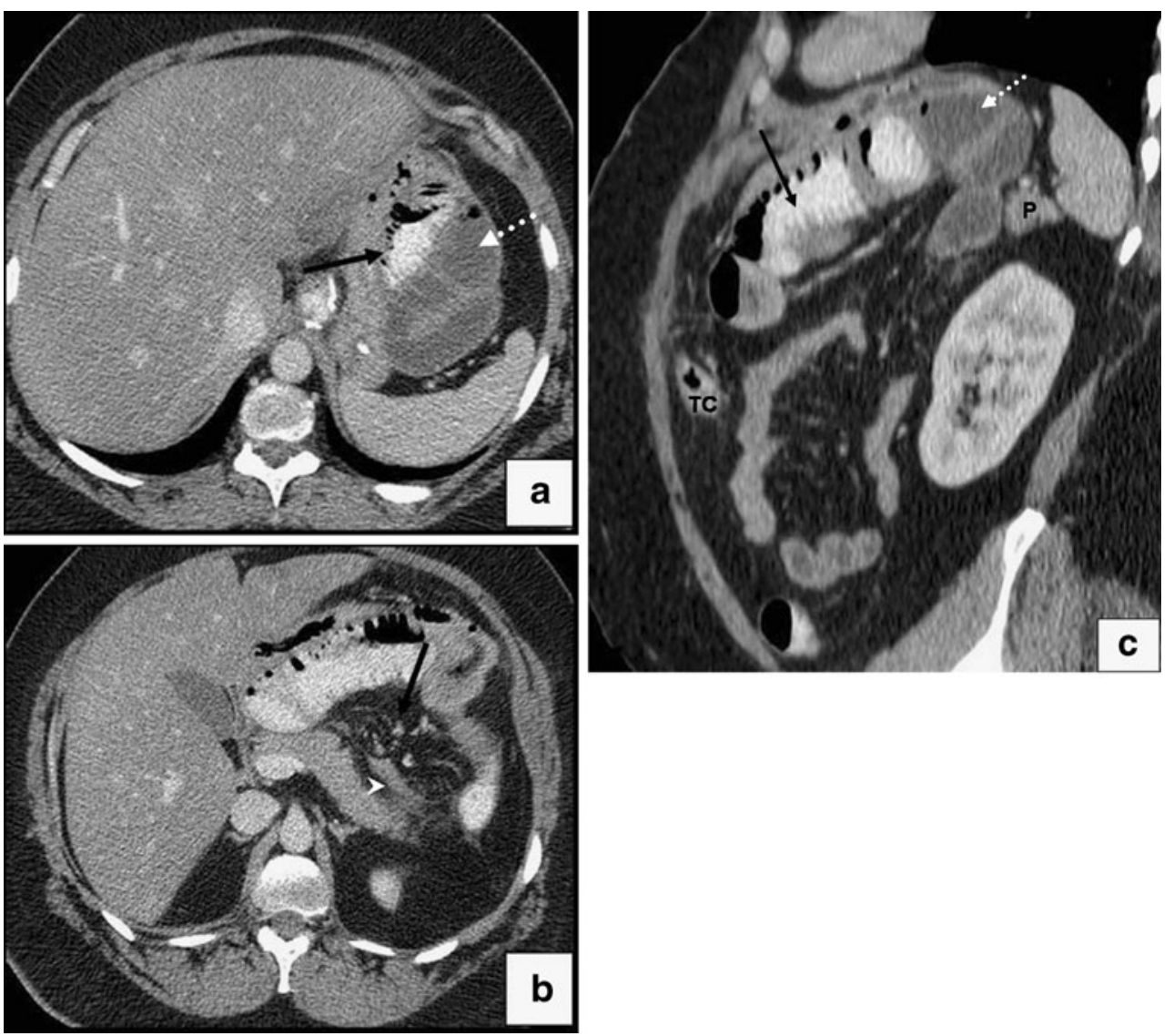

Fig. 5 Petersen's hernia in a 38-year-old woman occurring 20 months after LRYGBP. Axial $(\mathbf{a}, \mathbf{b})$ and coronal (c) MDCT images demonstrate the distension of the excluded stomach (a, dotted arrow) as well as a cluster of dilated small bowel loops (b, c, white arrowheads) abutting against the left abdominal wall and located at the left side of the Roux limb (a, black arrow; c, white arrow). Note the horizontal course of the superior mesenteric vessels (b, black arrowhead)
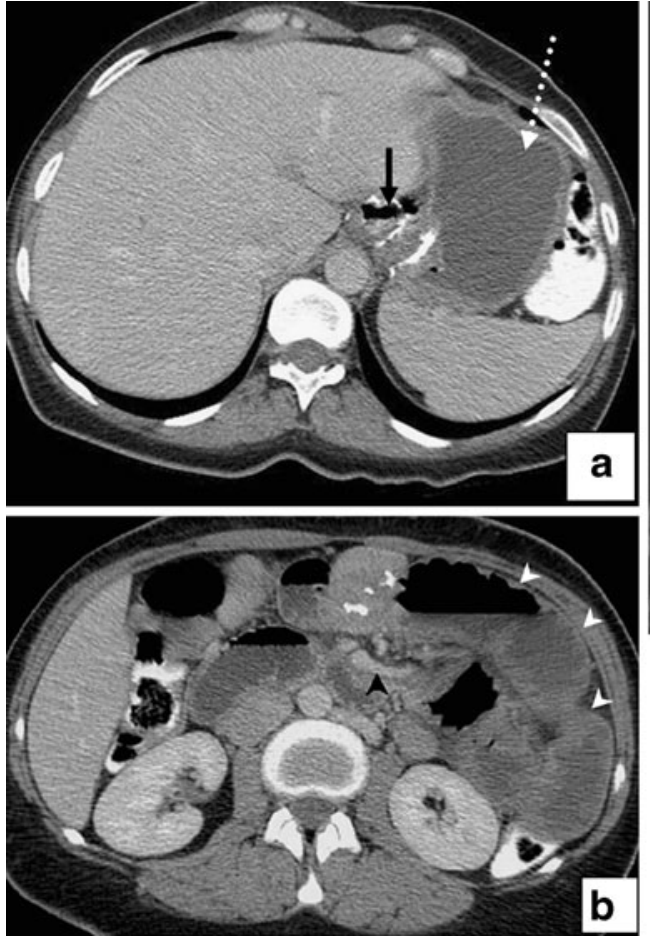

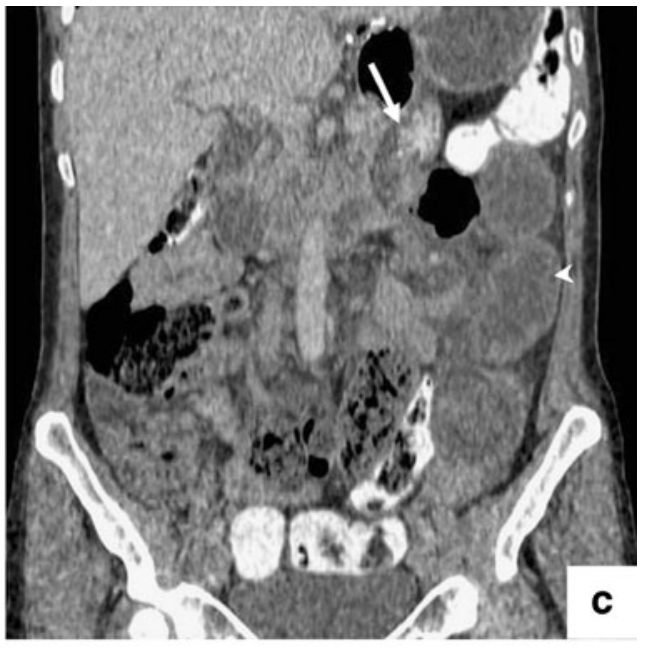


Fig. 6 Mesojejunal hernia occurring in a 32-year-oldwoman 2 years after LRYGBP. Axial CT images $(\mathbf{a}, \mathbf{b})$ nicely demonstrate the important dilatation of the excluded stomach (a, arrow) and of the Roux limb. Note the mesenteric whirl sign with subtle vascular deviation to the right abdomen $(\mathbf{b}$, dotted arrow) located near the jejunojejunal anastomosis indicating mechanical obstruction with volvulus, even better visible on coronal (c) MIP reconstructions
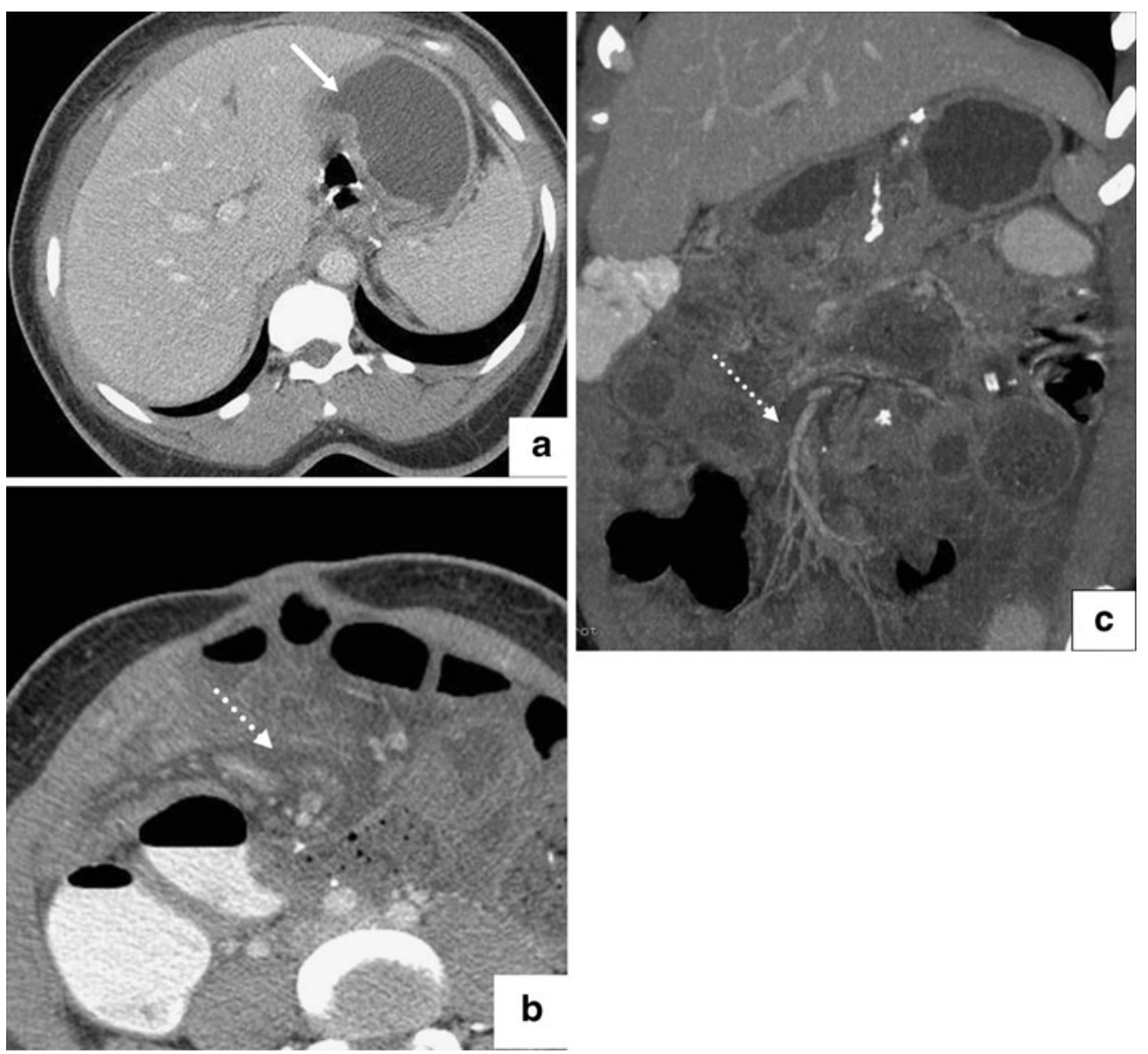

b different types [17]. The recognition of the anatomical abdominal landmarks and the direct visualization of the $\mathrm{IH}$ orifices are impossible on plain abdominal radiographs.

Furthermore, unlike MDCT, SBFT does not allow the straightforward detection of other postoperative complications, such as bleeding or bowel perforation. Thus, MDCT has been increasingly used for these complicated cases [3, 11], and it has even proven to be the single most effective radiological investigation for diagnosing IH post-LRYGB [10]. However, no attempt has yet been made to distinguish the four different types of IH by MDCT.

\section{SBO and $\mathrm{IH}$}

Although we deliberately limited our analysis on MDCT examinations performed in emergency on patients with acute abdominal pain immediately before surgery, IH were in our study population, as previously published [3-5, 8, 12, 17], only inconsistently associated with signs of SOB. The latter were seen in 25 (73.5\%) of our 34 patients only, probably because SBO intermittently occurred, or because the abdominal pain resulted from another undisclosed cause. The prevalence of SBO was similar in each group of $\mathrm{IH}$, suggesting that neither of them is more prone to induce SBO. Therefore, the radiological differentiation of $\mathrm{IH}$ must rely on other features than SBO.

In contradistinction to previous publications (Table 1), our study demonstrated that the dilatation of the remnant stomach or the Roux limb may be encountered in any of the four different types of IH. This is explained by the fact that these segments are always localized proximal to the incarcerated jejunal segment regardless of the type of the IH. Therefore, their dilatation only depends on the degree and the duration of associated SBO.

According to certain authors, the mesenteric whirl sign suggesting small bowel volvulus is the best indicator of $\mathrm{IH}$ after LRYGBP, unlike SBO, clustered small bowel loops, and vascular displacement. It also yields substantial interobserver agreement, unlike other signs [5, 9]. In our study population, the mesenteric whirl sign was seen in 22 (64.7\%) patients without permitting the distinction between the four types of $\mathrm{IH}$.

\section{Differentiation Between the Four Types of $\mathrm{IH}$}

The location of a transition point corresponding to the hernial orifice and the site of a sac-like cluster of small bowel loops, as well as the course of the vascular engorgement and crowding, may point to the anatomical site of $\mathrm{IH}$, thus giving a clue for 
Fig. 7 Jejunojejunal hernia in a 49-year-old woman 4 years after LRYGBP. Axial $(\mathbf{a}, \mathbf{b})$ and coronal (c, d) MIP show clustered small bowel dilatation (white arrowheads) with a beak sign (d, white arrow) indicating the transition point of the SBO, that is nicely detected between the two radiopaque stapler lines (a-d, black arrows) defining the laterolateral jejunostomy. It corresponds to the hernial orifice. Note the vascular pedicle (b, c, white star) belonging to the clustered small bowel loops entering into the space between the two jejunal limbs, thus widening the two stapler lines
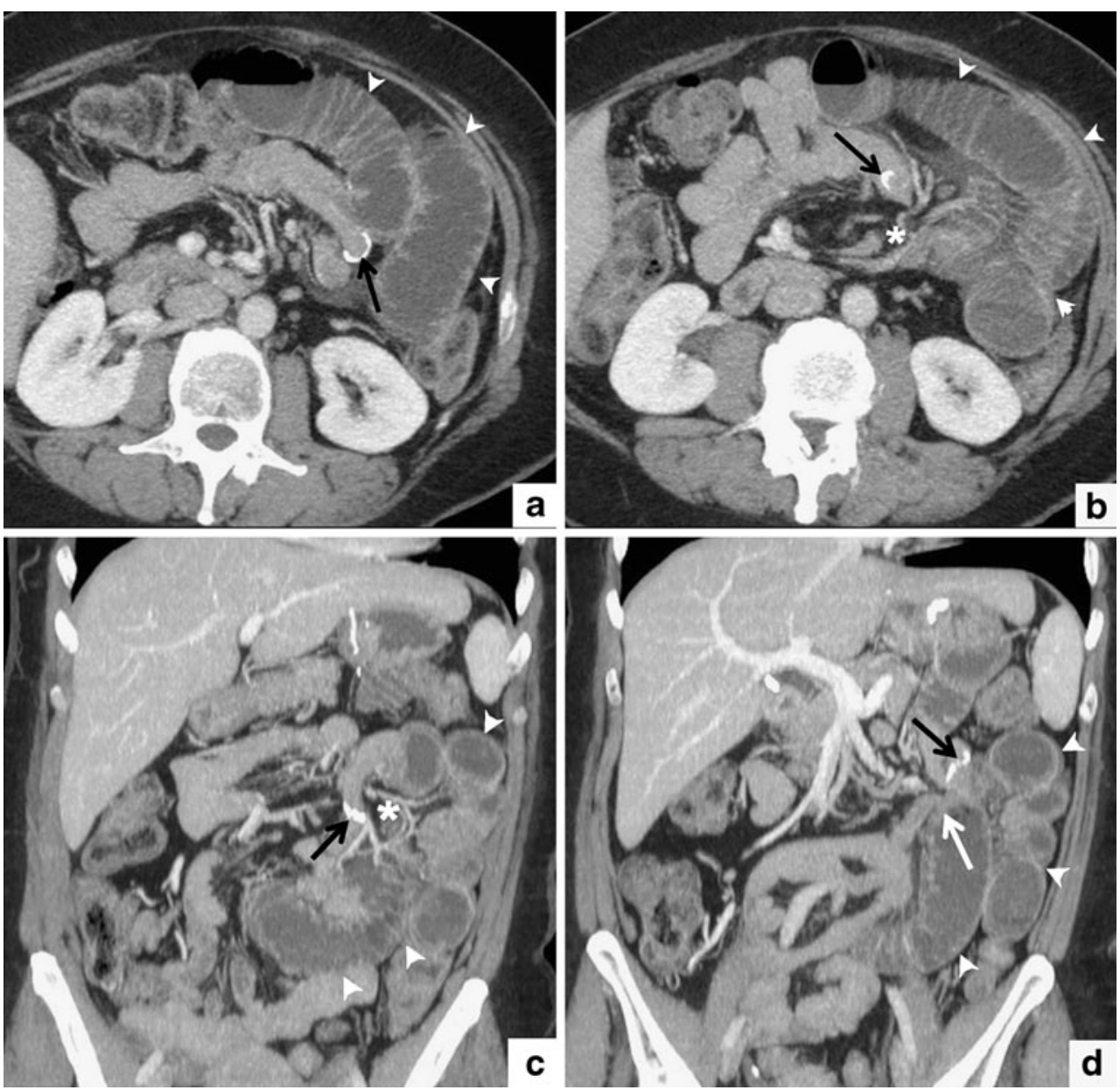

the differentiation between the four types. Our results indicate that the presence of clustered small bowel loops does not allow any distinction between the four $\mathrm{IH}$, but the difference in location helps identify two out of them, that are mesocolic and Petersen's IH. Carucci et al., evaluating SBFT for the different IH after RYGB, detected clustered small bowel loops in each of their 20 patients, however without any relation between the position and the anatomical type [17]. This may be due to the lesser anatomical landmarks available on plain abdominal radiographies compared to MDCT images. In our series, in only 29 out of 34 patients, a cluster of small bowel loops could be detected on MDCT, but their position was statistically significantly associated with a type of $\mathrm{IH}$.

Furthermore, Carucci et al. described the shift of the jejunojejunal anastomosis in all their SBFT studies with $\mathrm{IH}$, in which a radiopaque stapler line had been used, most often cephalad into the left upper quadrant [17]. In our series, this shift was only seen in $16(47 \%)$ patients and did not correlate with any type of $\mathrm{IH}$, unlike the widening of the jejunal anastomosis. The latter strongly indicates a jejunojejunal hernia $[6,13]$, the only type of $\mathrm{IH}$ for which radiological features had not been described yet, as to our knowledge.
Transmesocolic Hernia

In transmesocolic hernia, the small bowel prolapses through the surgically created window in the transverse mesocolon permitting the passage of the Roux limb (Figs. 2 and 3a). It cannot occur after antecolic placement of the Roux limb, thus avoiding the incision of the transverse mesocolon. The herniated bowel is usually the Roux limb itself with a variable amount of dilated small bowel segments, located anteriorly to the spleen and posteriorly to the stomach possibly exerting a mass effect on its posterior wall (Fig. 4a) and cephalic to the transverse mesocolon, which is displaced inferiorly. The transition point always lies proximal to the jejunojejunostomy, being typically situated at the level of the mesocolic window, nicely appreciated in the sagittal plane (Fig. 4c) [2, 3, 7, 10, 20, 21]. Some authors consider it the most common type of the four $\mathrm{IH}$ after LRYGBP [4, 7, 8, 17, 20, 23], while in our series, it was the least frequent one, although the majority of our patients had a retrocolic Roux limb. This difference may be explained by the increasing attention surgeons attach to the initial closure of this particular mesenteric defect during 
LRYGBP, since the mesocolic IH is the most discussed type in the recent surgical and radiological literature.

\section{Petersen's Hernia}

The Petersen's space is the opening between the transverse mesocolon posteriorly and the mesentery of the Roux limb anteriorly, caudally to the mesocolic defect created for the passage of the Roux limb (Figs. 2 and 3b). Thus, a Petersen's hernia (Fig. 5) means the presence of small bowel loops behind the Roux limb mesentery and anterior to the transverse mesocolon. Sometimes considered the second most common IH after the mesocolic type [4, 13], it has been found to be the most frequent $\mathrm{IH}$ in patients with antecolic antegastric RYGBP [24]. When small bowel obstruction is present, MDCT demonstrates a sac-like cluster of small bowel loops displaced into the left midabdomen, passing behind the Roux limb and in front of the Treitz angle, then abutting against the left mid-abdominal wall (Fig. 5b) [3, 21]. A horizontal course of the often engorged superior mesenteric vessels towards the left abdominal wall is seen (Fig. 6b). The latter two signs significantly indicated this type of IH in our study population. According to some authors, Petersen's hernia is not associated with any confining sac of intestinal loops $[7,20]$. However, in agreement with Yu et al. [3], we could identify a sac-like cluster of small bowel loops in each of our ten patients with this type of $\mathrm{IH}$, abutting against the left mid-abdominal wall in nine of our ten patients.

\section{Mesojejunal Hernia}

A mesojejunal hernia (Fig. 6) occurs when small bowel loops prolapse through the jejunojejunostomy mesenteric defect, a window between the two mesenteries belonging to the Roux limb and biliopancreatic limb that are joined at the distal anastomosis (Figs. 2 and 3c). As we demonstrated in mesojejunal $\mathrm{IH}$, a large cluster of dilated bowel segments, seen adjacent to the jejunojejunal anastomosis, is typically pressed against the anterior abdominal wall without overlying omental fat, with crowding and engorgement of the mesenteric blood vessels [7, 10]. In case of volvulus, the mesenteric whirl sign (Fig. 6b) is typically detected near the jejunojejunal anastomosis. The stomach (Fig. 6a) and/or the Roux limb may also be dilated $[9,11]$.

In agreement with two previous publications $[5,13]$, the mesojejunal hernia was the most common type out of the four IH in our study population. It should be suspected in case of a right anterolateral deviation of the SMV, occurring in seven patients $(p=0.0033)$, unlike the shift to the left abdomen seen in Petersen's IH. This may be explained by the more distal and also anterior location of this $\mathrm{IH}$ compared to the mesocolic and Petersen type.
Jejunojejunal Hernia

The jejunojejunal hernia (Fig. 7) has only recently been described as a complication after LRYGBP [6]. It is exactly located at the site of the laterolateral jejunojejunostomy, where small bowel loops enter into the interjejunal space that has been created between the staple line at the end of the biliopancreatic limb and the suture adjacent to the jejunojejunostomy (Figs. 2 and $3 \mathrm{~d}$ ). Since there is no mesenteric involvement, it cannot result from rapid weight loss, but the technique of surgery (the use of absorbable sutures or a remaining gap between single stitches) is the principal cause. Even very small surgical defects at this site may lead to the development of an internal hernia [6], with varying degrees of small bowel distension. However, it is always located around the jejunojejunal anastomosis, which is involved and where you may see the transition point in case of significant SBO. In our population, the incidence of jejunojejunal IH significantly correlated with the widening of the space between the closure of the afferent limb and the jejunostomy, seen in all of the five patients with this type of IH (Fig. 7). The three patients with mesojejunal IH, in whom we also detected this MDCT feature, had associated volvulus. Thus, the important mechanical distortion of the distal anastomosis may explain this feature.

\section{Limitations of the Study}

Our study has several limitations due to its retrospective patients' inclusion despite the use of the large, prospectively collected database. We used consensus reading rather than independent image analysis that would have allowed for inter- and intraobserver variations. Since we considered the semiology of IH as complex and necessary to be clarified during the reading process, consensus reading seemed to us more appropriate for this study.

Finally, our study population was composed of patients admitted in emergency for acute abdominal symptoms. Thus, the incidence of radiological signs of SBO may be modified, compared to patients with IH and less severe clinical symptoms.

\section{Conclusion}

In conclusion, our study demonstrates that none of the four IH occurring after LRYGBP seems associated with a higher risk of SBO. Careful attention to the anatomical landmarks as well as the identification and the position of clustered small bowel loops and the hernial orifice allows for the differentiation between the four types of IH. This may permit subsequent straightforward laparoscopic exploration. 
Acknowledgments We would like to thank Mr. Kevin Decitre for the drawings. Special thanks need to be expressed to Professor Marcel Hoogewood, head of the radiological department of the University Hospital Fribourg, Switzerland, as well as to Antoine Niklaus, chief radiologist of the regional hospitals of Aigle and Monthey, Switzerland, for providing patients and clinical information. Mr. Frédéric Pedron and Mr. Grégoire Haengartner helped in informatics devices.

Conflict of Interest None of the authors have anything to disclose, neither any commercial interest nor any source of financial or material support.

\section{References}

1. Balsiger BM, Murr MM, Poggio JL, et al. Bariatric surgery. Surgery for weight control in patients with morbid obesity. Med Clin North Am. 2000;84:477-89.

2. Reddy SA, Yang C, McGinnis LA, et al. Diagnosis of transmesocolic internal hernia as a complication of retrocolic gastric bypass: CT imaging criteria. AJR. 2007;189:52-5.

3. Yu J, Turner MA, Cho S-R, et al. Normal anatomy and complications after gastric bypass surgery: helical CT findings. Radiology. 2004;231:753-60.

4. Iannelli A, Facchiano E, Gugenheim J. Internal hernia after laparoscopic Roux-en-Y gastric bypass for morbid obesity. Obes Surg. 2006;16:1265-71.

5. Iannuccilli JD, Grand D, Murphy BL, et al. Sensitivity and specificity of eight $\mathrm{CT}$ signs in the preoperative diagnosis of internal mesenteric hernia following Roux-en-Y gastric bypass surgery. Clin Radiol. 2009;64:373-80.

6. Paroz A, Calmes JM, Romy S, et al. A new type of internal hernia after laparoscopic Roux-en-Y gastric bypass. Obes Surg. 2009;19 (4):527-30.

7. Blachar A, Federle MP, Pealer KM, et al. Gastointestinal complications of laparoscopic Roux-en-Y gastric bypass surgery: clinical and imaging findings. Radiology. 2002;223(3):625-32.

8. Higa KD, Ho T, Boone KB. Internal hernias after laparoscopic Roux-en-Y gastric bypass: incidence, treatment and prevention. Obes Surg. 2003;13:350-4.

9. Lockhart ME, Tessler FN, Canon CL, et al. Internal hernia after gastric bypass: sensitivity and specificity of seven CT signs with surgical correlation and controls. AJR. 2007;188:745-50.
10. Ahmed RA, Rickards G, Johnson J, et al. Radiological findings in symptomatic internal hernias after laparoscopic gastric bypass. Obes Surg. 2009;19:1530-5.

11. Onopchenko A. Radiological diagnosis of internal hernia after Roux-en-Y gastric bypass. Obes Surg. 2005;15:606-11.

12. Iannelli A, Burratti MS, Novellas $\mathrm{S}$, et al. Internal hernia as a complication of laparoscopic Roux-en-Y gastric bypass. Obes Surg. 2007; 17:1283-6.

13. Paroz A, Calmes JM, Giusti V, et al. Internal hernia after laparoscopic Roux-en-Y gastric bypass for morbid obesity: a continuous challenge in bariatric surgery. Obes Surg. 2006;16:1482-7.

14. Gunabushanam G, Shanka S, Czerniach DR, et al. Small-bowel obstruction after laparoscopic Roux-en-Y gastric bypass surgery. J Comput Assist Tomogr. 2009;33(3):369-75.

15. Ahmed AR, Rickards G, Husain S, et al. Trends in internal hernia incidence after laparoscopic Roux-en-Y gastric bypass. Obes Surg. 2007;17:1563-6.

16. Scheirey CD, Scholz F, Shah PC, et al. Radiology of the laparoscopic Roux-en-Y gastric bypass procedure: conceptualization and precise interpretation of results. RadioGraphics. 2006;26(5):1355-71.

17. Carucci LR, Turner MA, Shaylor SD. Internal hernia following Roux-en-Y gastric bypass surgery for morbid obesity: evaluation of radiographic findings at small-bowel examination. Radiology. 2009;251:762-70.

18. Merkle EM, Hallowell PT, Crouse C, et al. Roux-en-Y gastric bypass for clinically severe obesity: normal appearance and spectrum of complications at imaging. Radiology. 2005;234(3):674-83.

19. Martin LC, Merkle EM, Thompson WM. Review of internal hernias: radiographic and clinical findings. AJR. 2006;186:703-17.

20. Sandrasegaran K, Rajesh A, Lall C, et al. Gastrointestinal complications of bariatric Roux-en-Y gastric bypass surgery. Eur Radiol. 2005;15:254-62.

21. Patel RY, Baer JW, Texeira J, et al. Internal hernia complications of gastric bypass surgery in the acute setting: spectrum of imaging findings. Emerg Radiol. 2009;16(4):283-9.

22. Higa KD, Boone KB, Ho T. Complications of the laparoscopic Roux-en-Y gastric bypass: 1040 patients - what have we learned? Obes Surg. 2000;10:509-13.

23. Garza EJ, Kuhn J, Arnold D, et al. Internal hernias after laparoscopic Roux-en-Y gastric bypass. Am J Surg. 2004;188:796-800.

24. Bauman RW, Pirrello JR. Internal hernia at Petersen's space after laparoscopic Roux-en-Y gastric bypass: $6.2 \%$ incidence without closure - a single surgeon series of 1047 cases. Surg Obes Relat Dis. 2009;5(5):565-70. 